\title{
Ascites regression following neoadjuvant chemotherapy in prediction of treatment outcome among stage IIIc to IV high-grade serous ovarian cancer
}

Xia Xu' ${ }^{1}$, Fei Deng ${ }^{2}$, Mengmeng Lv², Binhui Ren ${ }^{3}$, Wenwen Guo ${ }^{4}$ and Xiaoxiang Chen ${ }^{2^{*}}$

\begin{abstract}
Background: No consensus exists on the outcome-related factors of interval debulking surgery (IDS) in patients with advanced high-grade serous ovarian cancer (HG-SOC) who underwent neoadjuvant chemotherapy (NAC). This study aimed to explore the optimal timing for IDS and the prognosis-associated factors of International Federation of Gynecology and Obstetrics stage IIIc to IV HG-SOC patients.

Methods: A total of 160 IIIc to IV stage HG-SOC patients were retrospectively analyzed. Patients with large volume ascites underwent NAC and subsequent IDS from the Jiangsu Institute of Cancer Research between 1993 and 2013. The outcome of IDS-associated factors was explored by logistic regression. To predict IDS outcome, the potential values of serum CA-125 levels and CA-125 decreasing kinetics were determined by the receiver operating characteristic curve. The associations between survival durations and covariates were assessed by Cox proportional hazards model and log-rank test.

Results: Optimal IDS was achieved in $80.6 \%$ of HG-SOC patients who underwent NAC. Multivariate analyses revealed that ascites regression ( $p=0.01)$, serum CA-125 level $(p=0.02)$, and CA-125 decreasing kinetics $(p=0.01)$ were independent optimal IDS predictors. CA-125 decreasing kinetics, IDS outcome, and ascites volume were independently associated with overall survival (OS) ( $p=0.04, p<0.01, p=0.03$, respectively) and progression-free survival (PFS) ( $p<0.01, p<0.01, p=0.02$, respectively). Patients who exhibited disappearance of ascites ( $<500 \mathrm{ml})$ had longer PFS (19.7 months vs.14.9 months) and OS (32.1 months vs. 26.0 months) than patients who exhibited residual ascites $(\geq 500 \mathrm{ml})$. Subsets with higher CA-125 decreasing kinetics $(\geq 2.2)$ had longer PFS (21.4 months vs.13. 1 months) and OS (29.6 months vs.26.8 months) than counterparts (kinetics $<2.2)$.
\end{abstract}

Conclusions: Ascites regression and CA-125 decreasing kinetics were independently associated with surgical outcome and prognosis in advanced HG-SOC patients who underwent NAC.

Keywords: High grade serous ovarian cancer, CA-125, Ascites, Primary debulking surgery, Interval debulking surgery

\footnotetext{
* Correspondence: cxxxxcyd@gmail.com

2Department of Gynecologic Oncology, Jiangsu Cancer Hospital, 42\#

Baiziting street, Nanjing, Jiangsu 210009, People's Republic of China

Full list of author information is available at the end of the article
} 


\section{Background}

Epithelial ovarian cancer (EOC) is the most lethal gynecological cancer in North America, Western Europe, and China [1, 2]; its mortality rate has insignificantly decreased since the 1970s [3]. A total of 41,516 EOC cases were registered in China in 2011[4]. In 2015, EOC resulted in an estimated 21,290 cases and 14,180 deaths in the United States [5].

EOC is a heterogeneous tumor group, with high-grade serous ovarian cancer (HG-SOC) as the archetype and main cause of the high fatality rate [6]. The treatment outcome of HG-SOC is very poor because this disease is most commonly diagnosed in advanced stages at initial treatment. The current standard therapeutic strategy for advanced ovarian cancer is maximum primary debulking surgery (PDS) followed by frontline taxane plus carboplatin chemotherapy. Cytoreduction aims to achieve optimal debulking, as the amount of residual tumor after this procedure is one of the most important prognostic factors for the survival of women with HG-SOC. Over the past 30 years, the definition of optimal cytoreduction has changed from residual tumors $<1-2 \mathrm{~cm}$ to absent macroscopic disease [7-11].

An optimal surgical procedure is not always possible for patients with advanced-stage HG-SOC (IIIc to IV) with poor performance status or medical contraindications. Morever, extensive internal organ resection and major blood loss are associated with a high risk of morbidity. To reduce the patient's tumor burden and improve therapeutic effect by allowing further surgery, surgeons investigated the possibility of reducing tumor size with two to four cycles of neoadjuvant chemotherapy (NAC). No consensus exists on the optimal timing indicators for interval cytoreduction surgery (IDS) in advanced ovarian cancer, including tumor markers, ascites volume, imaging information, and patients' status [12, 13].

Women who have a high perioperative risk profile or a low likelihood of achieving cytoreduction to $<1 \mathrm{~cm}$ (ideally to no visible disease) were suggested to receive NAC. Chemotherapy may increase the ratio of patients suitable for IDS; the rates of optimal resection in IDS after induction chemotherapy had been reported to range from 77 to 94\% [14-19]. Patients who underwent IDS after NAC had lower morbidity, lower requirement of intensive care unit admission, lower duration of hospital stay, and higher quality of life than the patients who underwent PDS.

A consensus exists that a complete resection of all macroscopic disease (at PDS or IDS) is an independent predictor of progression-free survival (PFS) and overall survival (OS). Unlike the advantages for resectability and response rates demonstrated by most studies, there is no evidence of survival advantage demonstrating that NAC followed by IDS is inferior to the gold standard procedure, and the role of NAC has been debated for years $[20,21]$. Different EOC subtypes have distinct clinical characteristics and prognosis [6, 22-25]. Furthermore, different research methods utilized distinct recruitment standards that varied from one to another in terms of ascites volume, gross tumor burden, and the International Federation of Gynecology and Obstetrics (FIGO) stage. No published comparative study currently exists on these issues in HG-SOC. Therefore, evaluating the value of platinum-based NAC in a large HG-SOC sub-population is urgently needed.

In the present study, we retrospectively analyzed clinicopathological factors in patients with HG-SOC who received NAC/IDS at the Jiangsu Institute of Cancer Research (JICR, PRC).

\section{Methods}

\section{Study population}

A retrospective chart review was conducted to identify all patients diagnosed with HG-SOC treated at the JICR from January 1, 1993 to December 31, 2013. The analysis included all the patients treated at the institution during that period and who met the recruitment standards.

Multidisciplinary team (MDT) consists of two gynecologic oncologists, two pathologists, one radiologist and one medical oncologist was set to indentify women with a high perioperative risk profile or a low likelihood of achieving optimal cytoreduction should receive NAC. Before NAC is delivered, 76 patients have histologic confirmation of an invasive ovarian, fallopian tube, or peritoneal cancer from core biopsy. In exceptional cases, when a biopsy cannot be performed, cytologic evaluation combined with a serum CA-125 to carcinoembryonic antigen (CEA) ratio $>25$ is acceptable to confirm the primary diagnosis in 62 cases. In other 22 cases with CA125 to CEA ratio was 25 or lower, patients had to have an additional barium enema or colonoscopy, gastroscopy or radiologic examination of the stomach, and mammography to rule out a potential metastatic primary malignancy. Under these criteria, the incidence of other malignancies confirmed by IDS was 5\%; and only 8 patients were excluded in further analysis.

The recruit criteria of the present study were as follows: patients must be histological confirmed stage IIIc to IV ovarian, fallopian tube, or peritoneal high-grade serous cancer by IDS; An additional inclusion criterion of large volume ascites estimated by ultrasound and confirmed by surgical procedure was set to observe the regression of ascites by NAC (35 frailty cases underwent slow-release evacuation procedure before NAC treatment for the intolerable abdominal distension were excluded); patients who underwent PDS were excluded; patients who underwent non-platinum NAC were 
excluded; patients who did not undergo entire primary therapy procedure were excluded; patients with preoperative serum CA-125 levels $<=35 \mathrm{U} / \mathrm{ml}$ were excluded; laparoscopic proved HG-SOCs were excluded. The number of NAC cycles was administered based on the MDT's decision. During 1993-1997, cisplatin $\left(50 \mathrm{mg} / \mathrm{m}^{2}\right)$, farmorubicin $\left(50 \mathrm{mg} / \mathrm{m}^{2}\right)$, and a cyclophosphamide $\left(500 \mathrm{mg} / \mathrm{m}^{2}\right)$ combined regime was administrated to 18 HG-SOCs every 3 weeks. After 1997, 142 cases underwent carboplatinum (area under the curve 5-6) and paclitaxel $\left(135-175 \mathrm{mg} / \mathrm{m}^{2}\right)$ regime every 3 weeks. An IDS was scheduled approximately 2 to 4 weeks after NAC. Patients were then treated with at least three to four additional chemotherapy cycles with the same regimen as NAC. Disease progression was evaluated by computed tomography (CT, all cases)/magnetic resonance imaging (MRI, 49 cases) or positron emission tomography imaging (PET/CT, 25 cases) of the abdomen and pelvis before initiating of frontline or second-line chemotherapy. The patients' follow-up plan after the completion of primary treatment included clinical assessment and serum marker measurement, as mentioned previously [22-24].

\section{Clinicopathological characteristics}

The clinicopathological data of the patients were reviewed and the following data were collected: age; tumor grade; histology; tumor stage; serum CA-125 and CEA levels during diagnosis, therapy, and follow-up; NAC regime, courses, and clinical or pathological responses; optimality of cytoreductive surgery; and disease status at the last follow-up. Ascites volume was estimated by ultrasound and confirmed by surgical procedure. Regression was defined by as an ascites volume $<500 \mathrm{ml}$. CA-125 decreasing kinetics was defined as the ratio of the initial serum CA-125 level divided by the preoperative serum CA-125 level. Surgical staging followed the FIGO system. Optimal cytoreduction was defined as the absence of macroscopic disease on the completion of the surgical procedure.

OS was defined as the length of time from diagnosis to death, or to the last follow-up examination of patients who are still alive. PFS was defined as the time interval from primary treatment where in the patient's condition did not worsen. Clinical response of administration, including NAC, IDS, and adjuvant chemotherapy, was defined according to the standards of the Response Evaluation Criteria in Solid Tumors [26]. The pathology of all patients was initially reviewed by pathologists from JICR (Hou and $\mathrm{Xu}$ ). A panel of pathologic markers was routinely measured. This study was approved by the ethics committee of the Jiangsu Institute of Cancer Research. Informed consent was obtained from all involved participants.

\section{Statistical analysis}

The association between survival and adjuvant chemotherapy regime, courses, and therapy response was assessed by the Cox proportional hazards model. The multivariate model was constructed by step-wise regression techniques. $P$-values of less than 0.05 were considered to be significant. Survival distributions were estimated by the Kaplan-Meier method, and statistical significance was determined by log-rank test and Cox's proportional hazard model analysis. Optimal IDS-related potential factors were explored by logistic regression analysis. All data manipulation and statistical analysis were performed by SPSS software v16 (SPSS for Windows, Rel.16. Chicago: SPSS Inc.).

\section{Results}

\section{Patient characteristics}

Among the 382 patients with advanced stage HGSOC with large volume of ascites underwent entire primary therapy procedure in JICR, 160 (41.9\%) patients received NAC and IDS, meeting the study criteria; 129 (80.1\%) patients received subsequent optimal debulking surgery; and 31 patients received suboptimal debulking surgery (macroscopic residual disease). The median number of NAC cycles was 3 (range, 2 to 4). The clinicopathological characteristics of recruited cases are described in Table 1. The median follow-up duration of the survivors was 45 months (interquartile range, 38.2 to 56.4 months).

Table 1 Patient characteristics of the study population

\begin{tabular}{lll}
\hline Characteristic & Median & Percentage/range \\
\hline Age (years) & 62.2 & $36-82$ \\
Initial CA-125 level (U/mL) & 920 & $41-24440$ \\
CA-125 decreasing kinetics & 2.2 & $0.9-28.6$ \\
Tumor sites ${ }^{\text {a }}$ & & \\
$\quad<=3$ & 38 & $23.7 \%$ \\
$>3$ & 122 & $76.3 \%$ \\
Preoperative ascites & & \\
$<500$ ml & 88 & $55.0 \%$ \\
$>=500$ ml & 72 & $45.0 \%$ \\
Surgical residual & & \\
No macroscopic focus & 129 & $80.6 \%$ \\
$1-2$ cm & 6 & $3.8 \%$ \\
$>2$ cm & 23 & $14.4 \%$ \\
Unknown & 2 & $1.3 \%$ \\
FIGO stage ${ }^{b}$ & & \\
IIIc & 133 & $83.1 \%$ \\
IV & 27 & $16.9 \%$ \\
\hline Tum sies a abdom is & &
\end{tabular}

Tumor sites ${ }^{a}$ abdomen is divided into four quadrants according to the belly button

$\mathrm{FIGO}^{\mathrm{b}}$ the International Federation of Gynecology and Obstetrics 


\section{Predictors of optimal IDS}

The optimal IDS subset had a median preoperative serum CA-125 level of $445 \mathrm{U} / \mathrm{mL}$ (range, $45-730 \mathrm{U} / \mathrm{mL}$ ) and CA-125 decreasing kinetics of 2.8. The suboptimal IDS group had a median preoperative serum CA-125 of $653 \mathrm{U} / \mathrm{mL}$ (range, 41-980 U/mL) and CA-125 decreasing kinetics of 1.7 .

Univariate logistic regression analysis revealed that ascites regression, serum CA-125 level and decreasing kinetics, tumor sites, and patient's age and status were IDS outcome-related factors (Table 2). Furthermore, we found that ascites regression $(p=0.01)$, preoperative serum CA-125 level $(p=0.02)$, and CA-125 decreasing kinetics $(p=0.01)$ were independent optimal IDS predictors in multivariate analyses (Table 2). Analysis with the receiver operating characteristic (ROC) curve revealed that CA-125 decreasing kinetics $(p=0.02)$ and preoperative ascites volume $(p<0.01)$ might imply optimal IDS (Fig. 1a and b).

\section{Ascites regression was associated with prognosis}

Using the univariate Cox proportional hazards model, we found that OS and PFS were associated with IDS outcome $(p<0.01$ and $p<0.01$, respectively), preoperative serum CA-125 level $(p=0.02$ and $p=0.04$, respectively), CA-125 decreasing kinetics $(p<0.01$ and $p<0.01$, respectively), and ascites volume $(p<0.01$ and $p<0.01$, respectively) as shown in Table 3 . Multivariate analysis revealed that OS and PFS were independently associated with CA-125 decreasing kinetics $(p=0.04$ and $p<0.01$, respectively), IDS outcome $(p<0.01$ and $p<0.01$, respectively), and ascite volume $(p=0.03$ and $p=0.02$, respectively) as shown in Table 3.

Ascites regression indicated longer OS durations (32.1 months, 95\% confidence interval [CI] 27.1-37.1 vs. 26.0 months, [CI] 22.3-29.6, Fig. 2a) and PFS (19.7 months, [CI] $17.8-21.6$ vs. 14.9 months, [CI] 12.517.2, Fig. 2b) for patients who underwent IDS. We set the cut-off point at 2.2 for CA-125 decreasing kinetics. Groups with higher CA-125 decreasing kinetics had

Table 2 Logistic regression of optimal IDS associated factors in advanced HG-SOCs

\begin{tabular}{lccccc}
\hline Factors & \multicolumn{2}{c}{ Univariate } & & \multicolumn{2}{c}{ Multivariate } \\
\cline { 2 - 3 } & $\operatorname{Exp}(\beta)$ & Sig & & $\operatorname{Exp}(\beta)$ & Sig \\
\hline Age & 1.01 & 0.15 & & 1.00 & 0.52 \\
Ascites & 1.90 & 0.01 & & 2.20 & 0.01 \\
Stage & 1.4 & 0.30 & & 1.33 & 0.18 \\
Tumor sites & 1.3 & 0.22 & & 1.16 & 0.47 \\
Baseline CA-125 & 1.13 & 0.01 & & 1.08 & 0.35 \\
Preoperative CA-125 & 1.28 & 0.00 & & 1.05 & 0.02 \\
CA-125 decreasing kinetics & 2.15 & 0.01 & & 2.40 & 0.01 \\
\hline
\end{tabular}

higher OS (29.6 months, [CI] 26.1-33.0 vs. 26.8 months, [CI] 22.7-30.1) and PFS (21.4 months, [CI] 18.6-24.2 vs. 13.1 months, [CI] 10.8-15.5) durations than groups with lower CA-125 decreasing kinetics (Fig. 3a and $b$ ).

\section{Discussion}

Ovarian cancer is not a single disease entity, but rather comprises a heterogeneous group of tumors with distinct clinicopathological characteristics. Inconsistent outcomes regarding the prognostic significance of NAC may be related to the failure to consider tumor grade, histotype, stage, and chemotherapy response [27-32]. In the current study, we identified optimal IDS and prognosis-related factors in advanced HG-EOC patients who underwent NAC.

We suggested that patient stratification by ascites regression may be useful in analyzing the surgical timing of NAC in advanced HG-EOC. Based on our previous analysis [22-24], we assumed that CA-125 decreasing kinetics implied NAC response and followed IDS outcomes. Stratification by serum CA-125 level and CA-125 decreasing kinetics revealed that NAC group patients with preoperative serum CA-125 levels $<450 \mathrm{U} / \mathrm{ml}$ and decreasing kinetics $>2.2$ significantly benefited in PFS and OS. This result implied that only selected patients, such as patients with HG-SOC and higher serum CA125 levels and drastically reduced CA-125 kinetics, may receive survival benefit from NAC. Our data also suggested that biomarkers or other prediction models may be applied to identify target groups that can benefit from NAC. The most intriguing feature of the current data is the trend of the NAC hazard ratio according to the spectrum of preoperative ascites volume. Patients with a large amount of ascites had significantly poorer IDS outcomes compared with patients who exhibited ascites regression. This result suggests that NAC provides superior IDS outcome in a select subset of patients.

In the present cohort, optimal IDS rate was significantly higher in patients with CA-125 decreasing kinetics higher than 2.2 or ascites regression. Therefore, it was naturally expected that the improved optimal resection rate by NAC resulted in improved survival outcome. Reports had repeatedly stated that the improved optimal debulking rate obtained by NAC/IDS cannot directly translate into increased PFS or OS compared with PDS [33-36]. Although chemotherapy-assisted optimal cytoreduction is not biologically equivalent to optimal surgic al debulking, NAC also aims to increase the optimal resection rate. Our data proved that the patient subset exhibiting ascites regression had as uperior optimal resection rate. Therefore, ascites regression should be regarded as a timing indicator for IDS. Ascites regression may be associated with histotype, chemotherapy 

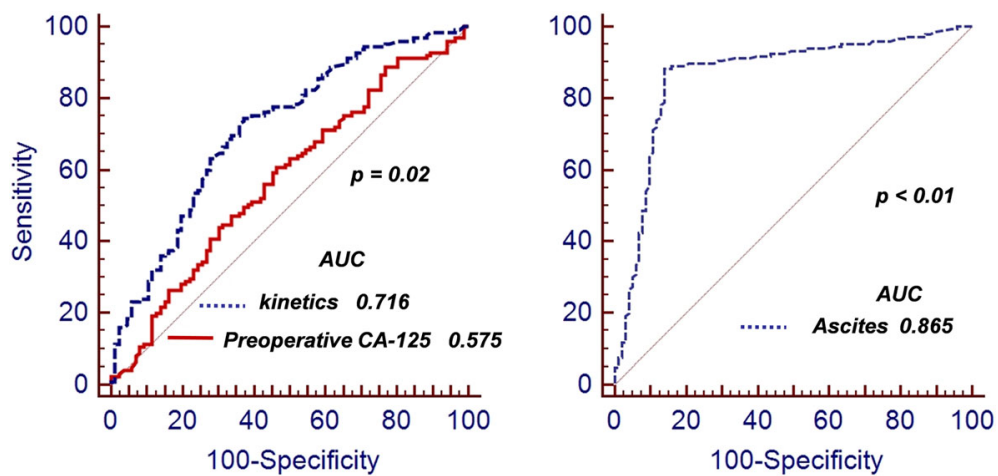

Fig. 1 ROC curve for the outcome of interval cytoreduction surgery by preoperative serum CA-125 level, decreasing kinetics, and ascites volume

response, tumor volume and sites, and even invasion depth [37-42]. The higher optimal resection rate and chemotherapy response rate accompany by the disappearance of ascites may explain the association between ascites regression and prognosis.

Up to present, there were four randomized clinical trials comparing PDS and NAC followed by IDS for women with advanced ovarian cancer [36, 43-45]. These trials demonstrated that NAC/IDS was noninferior to PDS with respect to PFS and OS and resulted in a lower incidence of treatment-related morbidity and mortality. In EORTC 55971 [36], residual tumor of $1 \mathrm{~cm}$ or less was achieved in $42 \%$ of patients in the PDS arm and in $81 \%$ of patients in the NAC/IDS arm. Cytoreduction to $<=1 \mathrm{~cm}$ residual disease was achieved in $41 \%$ of patients in the PDS arm and 73\% of patients in the NAC/IDS arm $(p=0.0001)$ in the CHORUS trial [43]. The SCORPION $[44,46]$ trial found that complete cytoreduction was achieved in $58 \%$ of women in the NAC/IDS arm and $46 \%$ of women in the PDS arm, with a shorter median operative time in the NAC/IDS arm. In JCOG0602 [45], Optimal debulking proportions in NAC/IDS and PDS were 82 and $37 \%$, respectively. Most of HG-SOCs are sensitive to platinum-based chemotherapy, and optimal debulking proportions increased in patients who underwent NAC. The rate of optimal cytoreduction in our study is up to $80.6 \%$, similar to existing trials. It is very unlikely that an optimal surgical cytoreduction can be achieved in all HG-SOC patients who underwent NAC from literatures to the present study.

Table 3 Survival-related characteristics in advanced HG-SOCs who underwent NAC/IDS

\begin{tabular}{|c|c|c|c|c|c|c|c|c|}
\hline \multirow[t]{2}{*}{ Variable } & \multicolumn{4}{|c|}{ Univariate } & \multicolumn{4}{|c|}{ Multivariate } \\
\hline & \multicolumn{2}{|l|}{ PFS } & \multicolumn{2}{|l|}{ OS } & \multicolumn{2}{|l|}{ PFS } & \multicolumn{2}{|l|}{ OS } \\
\hline \multicolumn{9}{|l|}{ FIGO stage } \\
\hline IV & 1.00 & (reference) & 1.00 & (reference) & 1.00 & (reference) & 1.00 & (reference) \\
\hline IIIC & 3.03 & $(0.35-13.66)$ & 4.24 & $(0.52-6.95)$ & 2.28 & $(0.30-4.10)$ & 3.57 & $(0.48-6.46)$ \\
\hline \multicolumn{9}{|l|}{ Ascites } \\
\hline$>=500 \mathrm{ml}$ & 1.00 & (reference) & 1.00 & (reference) & 1.00 & (reference) & 1.00 & (reference) \\
\hline$<500 \mathrm{ml}$ & 1.82 & $(1.45-2.85)$ & 1.90 & $(1.56-2.98)$ & 2.05 & $(1.40-2.95)$ & 2.02 & $(1.66-4.08)$ \\
\hline \multicolumn{9}{|l|}{ Outcome of surgery } \\
\hline Suboptimal & 1.00 & (reference) & 1.00 & (reference) & 1.00 & (reference) & 1.00 & (reference) \\
\hline Optimal & 1.52 & $(1.15-2.42)$ & 1.66 & $(1.22-2.50)$ & 1.93 & $(1.75-2.84)$ & 2.20 & $(1.40-4.24)$ \\
\hline \multicolumn{9}{|l|}{ Tumor sites } \\
\hline$>3$ & 1.00 & (reference) & 1.00 & (reference) & 1.00 & (reference) & 1.00 & (reference) \\
\hline$<=3$ & 1.32 & $(1.12-1.80)$ & 1.49 & $(1.25-2.25)$ & 1.26 & $(0.94-1.74)$ & 1.40 & $(0.85-2.04)$ \\
\hline Baseline CA-125 & 1.02 & $(1.01-1.08)$ & 1.03 & $(1.01-1.08)$ & 1.02 & $(0.97-1.07)$ & 1.02 & $(0.98-1.06)$ \\
\hline Preoperative CA-125 & 1.01 & $(1.00-1.04)$ & 1.01 & $(1.00-1.04)$ & 1.02 & $(0.96-1.05)$ & 1.02 & $(0.96-1.05)$ \\
\hline CA-125 decreasing kinetics & 1.02 & $(1.00-1.04)$ & 1.02 & $(1.00-1.04)$ & 1.01 & $(1.00-1.04)$ & 1.01 & $(1.00-1.05)$ \\
\hline
\end{tabular}



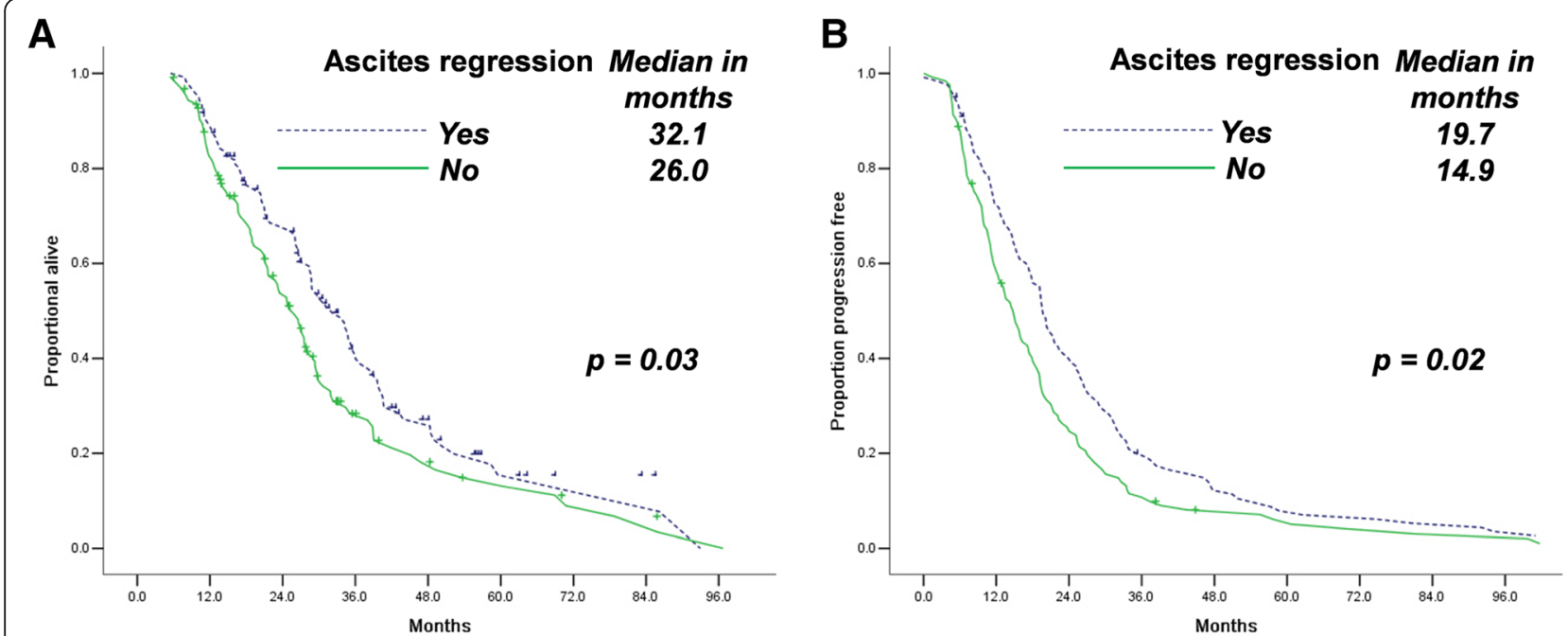

Fig. 2 Ascites regression $(\mathbf{a}, \mathbf{b})$ is associated with longer OS and PFS in patients with advanced stage HG-SOC who underwent NAC/IDS

This retrospective analysis has several limitations. Firstly, unavoidable selection biases are inherent to its design. Given the long durations of our study, the influence of the administration change such as the emergence of new chemotherapy and molecular target agents, the improvement of disease evaluate strategy is difficult to exclude. The relatively severe recruited criteria may partly eliminate the influence of selected factors. Secondly, the absence of unified recruited standard for NAC/IDS and limited sample size also causes bias though MDT was set to indentify cases. Last but not least, recruited advanced stage HG-SOCs who underwent NAC/IDS were relatively older and comparatively frail. It cannot be translated to all EOCs directly until studies with accurate inclusion and exclusion criteria are available. Overall, given the selection bias of this nonblinded study, the application of these favorable outcomes to all epithelial ovarian carcinoma patients should be with caution.

\section{Conclusions}

Our study supports the emerging evidence that ascites regression in advanced HG-SOCs is a predictor of optimal IDS. Ascites regression should be considered as an independent prognostic factor for both OS and PFS.
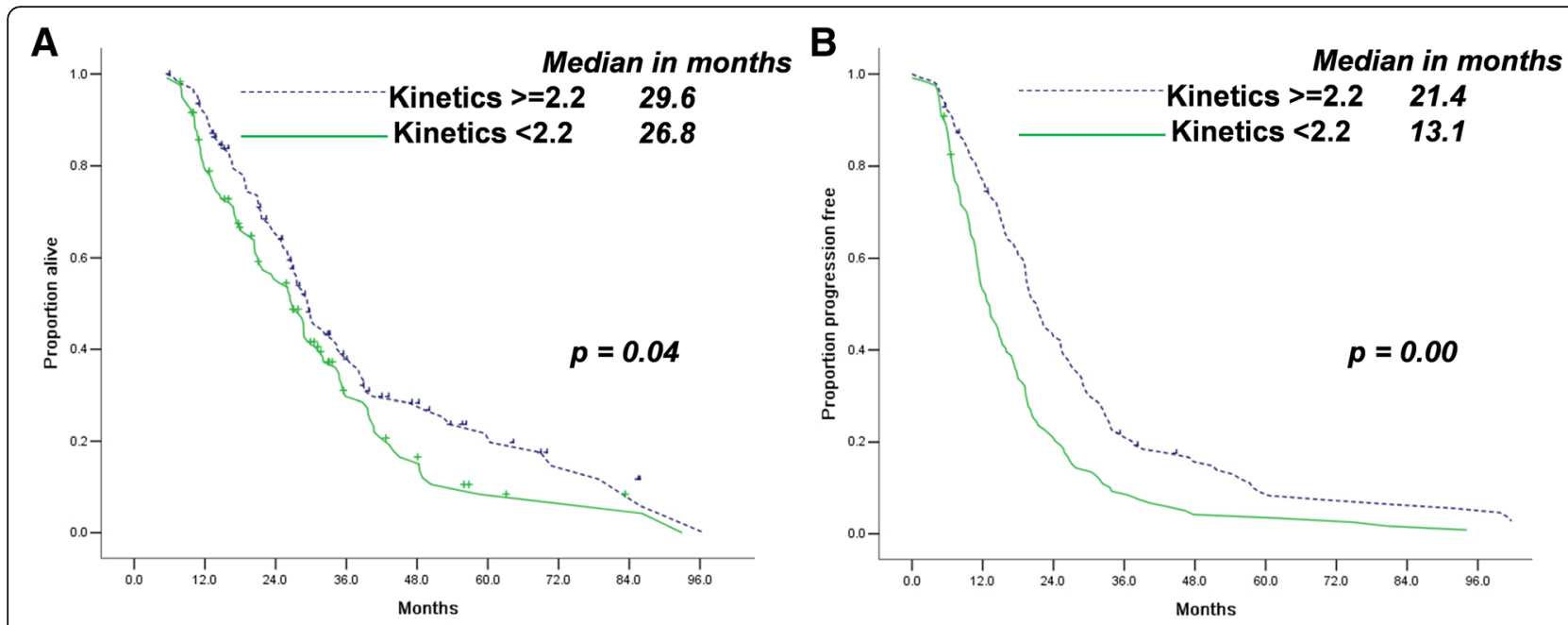

Fig. 3 Higher CA-125 decreasing kinetics $(\mathbf{a}, \mathbf{b})$ is associated with longer OS and PFS in patients with advanced HG-SOCs who underwent NAC/IDS 


\section{Abbreviations}

EOC: Epithelial ovarian cancer; IDS: Interval cytoreduction surgery; NAC: Neoadjuvant chemotherapy; OS: Overall survival; PDS: Primary debulking surgery; PFS: Progression free survival

\section{Acknowledgments}

Not applicable.

\section{Funding}

This study was supported by grants from the National Natural Science Foundation of China (No. 81472441, 81372321, 81672869), Natural Science Foundation of Jiangsu Province, China (No. BK20131439), six major talent summit (No. 2013-wsn-62).

\section{Availability of data and materials}

We would not share the data and material used in this manuscript, because we need them for further research.

\section{Authors' contributions}

XX participated in the design of present study and drafted the manuscript. FD carried out the cases recruit of present study. ML participated in the cases recruit of present study. BR carried out statistical analysis. WG participated in the statistical analysis and drafted the manuscript. XC designed of the study, performed the statistical analysis and revised the manuscript. All authors read and approved the final manuscript.

\section{Competing interests}

The authors declare that they have no competing interests.

\section{Consent for publication}

Not applicable.

\section{Ethics approval and consent to participate}

This retrospective study was approved by the institutional review board of Jiangsu Cancer Hospital, Nanjing Medical University, China. The informed consent requirement was waived. The committee's reference number was Jiangsu Cancer Hospital's Ethical Committee 2016-219.

\section{Author details}

'Department of Chemotherapy, Jiangsu Cancer Hospital, Nanjing, Jiangsu 210009, People's Republic of China. ²Department of Gynecologic Oncology, Jiangsu Cancer Hospital, 42\# Baiziting street, Nanjing, Jiangsu 210009, People's Republic of China. ${ }^{3}$ Department of Thoracic Oncology, Jiangsu Cancer Hospital, Nanjing, Jiangsu 210009, People's Republic of China. ${ }^{4} T$ The Second Affiliated Hospital of Nanjing Medical University, Nanjing, Jiangsu 210009, People's Republic of China.

\section{Received: 7 July 2016 Accepted: 29 November 2016}

\section{Published online: 02 December 2016}

\section{References}

1. Hannibal CG, et al. Survival of ovarian cancer patients in Denmark: excess mortality risk analysis of five-year relative survival in the period 1978-2002 Acta Obstet Gynecol Scand. 2008;87(12):1353-60

2. Yang L, et al. Statistics on cancer in China: cancer registration in 2002. Eur J Cancer Prev. 2005;14(4):329-35.

3. Bast Jr RC, Hennessy B, Mills GB. The biology of ovarian cancer: new opportunities for translation. Nat Rev Cancer. 2009;9(6):415-28.

4. Chen W, et al. Annual report on status of cancer in China, 2011. Chin Cancer Res. 2015;27(1):2-12.

5. Siegel RL, Miller KD, Jemal A. Cancer statistics, 2015. CA Cancer J Clin. 2015;65(1):5-29.

6. Chen $X$, et al. Cancer stem cells, epithelial-mesenchymal transition, and drug resistance in high-grade ovarian serous carcinoma. Hum Pathol. 2013; 44(11):2373-84

7. Hoskins WJ, et al. The effect of diameter of largest residual disease on survival after primary cytoreductive surgery in patients with suboptimal residual epithelial ovarian carcinoma. Am J Obstet Gynecol. 1994;170(4): 974-9. discussion 979-80.

8. Bristow RE, et al. Survival effect of maximal cytoreductive surgery for advanced ovarian carcinoma during the platinum era: a meta-analysis. J Clin Oncol. 2002;20(5):1248-59.
9. Elattar A, et al. Optimal primary surgical treatment for advanced epithelial ovarian cancer. Cochrane Database Syst Rev. 2011:8:CD007565.

10. Vergote I, Amant F, Leunen K. Neoadjuvant chemotherapy in advanced ovarian cancer: what kind of evidence is needed to convince US gynaecological oncologists? Gynecol Oncol. 2010;119(1):1-2.

11. Chi DS, et al. What is the optimal goal of primary cytoreductive surgery for bulky stage IIIC epithelial ovarian carcinoma (EOC)? Gynecol Oncol. 2006; 103(2):559-64.

12. Eltabbakh $\mathrm{GH}$, et al. Factors associated with cytoreducibility among women with ovarian carcinoma. Gynecol Oncol. 2004;95(2):377-83.

13. Angioli $\mathrm{R}$, et al. Can the preoperative HE4 level predict optimal cytoreduction in patients with advanced ovarian carcinoma? Gynecol Oncol. 2013:128(3):579-83.

14. Ansquer $Y$, et al. Neoadjuvant chemotherapy for unresectable ovarian carcinoma: a French multicenter study. Cancer. 2001:91(12):2329-34.

15. Kuhn W, et al. Neoadjuvant chemotherapy followed by tumor debulking prolongs survival for patients with poor prognosis in International Federation of Gynecology and Obstetrics Stage IIIC ovarian carcinoma. Cancer. 2001;92(10):2585-91.

16. Chan JK, et al. Stages III and IV invasive epithelial ovarian carcinoma in younger versus older women: what prognostic factors are important? Obstet Gynecol. 2003;102(1):156-61.

17. Morice $P$, et al. Results of interval debulking surgery in advanced stage ovarian cancer: an exposed-non-exposed study. Ann Oncol. 2003;14(1):74-7.

18. Giannopoulos T, et al. Clinical outcomes of neoadjuvant chemotherapy and primary debulking surgery in advanced ovarian carcinoma. Eur J Gynaecol Oncol. 2006;27(1):25-8

19. Lee $\mathrm{SJ}$, et al. Preliminary results of neoadjuvant chemotherapy with paclitaxel and cisplatin in patients with advanced epithelial ovarian cancer who are inadequate for optimum primary surgery. J Obstet Gynaecol Res. 2006;32(1):99-106.

20. Bristow RE, Chi DS. Platinum-based neoadjuvant chemotherapy and interval surgical cytoreduction for advanced ovarian cancer: a meta-analysis. Gynecol Oncol. 2006;103(3):1070-6.

21. Onda T, Yoshikawa H. Neoadjuvant chemotherapy for advanced ovarian cancer: overview of outcomes and unanswered questions. Expert Rev Anticancer Ther. 2011;11(7):1053-67.

22. $\mathrm{Xu} X$, et al. Secondary cytoreduction surgery improves prognosis in platinum-sensitive recurrent ovarian cancer. J Exp Clin Cancer Res. 2013;32:61

23. Chen $X$, et al. CA-125 level as a prognostic indicator in type I and type II epithelial ovarian cancer. Int J Gynecol Cancer. 2013;23(5):815-22.

24. Xu X, et al. Nadir CA-125 level as prognosis indicator of high-grade serous ovarian cancer. J Ovarian Res. 2013;6:31.

25. Wang F, et al. CA-125-indicated asymptomatic relapse confers survival benefit to ovarian cancer patients who underwent secondary cytoreduction surgery. J Ovarian Res. 2013;6(1):14

26. Therasse $P$, et al. New guidelines to evaluate the response to treatment in solid tumors. European Organization for Research and Treatment of Cancer, National Cancer Institute of the United States, National Cancer Institute of Canada. J Natl Cancer Inst. 2000:92(3):205-16.

27. Asada K, et al. Poor prognosis of ovarian cancer with large cell neuroendocrine carcinoma: case report and review of published works. J Obstet Gynaecol Res. 2014;40(3):869-72.

28. Chao KC, et al. Prognosis for advanced-stage primary peritoneal serous papillary carcinoma and serous ovarian cancer in Taiwan. Taiwan J Obstet Gynecol. 2013;52(1):81-4.

29. Hofstetter $\mathrm{G}$, et al. The time interval from surgery to start of chemotherapy significantly impacts prognosis in patients with advanced serous ovarian carcinoma - analysis of patient data in the prospective OVCAD study. Gynecol Oncol. 2013;131(1):15-20.

30. Jorgensen $T L$, et al. Significance of age and comorbidity on treatment modality, treatment adherence, and prognosis in elderly ovarian cancer patients. Gynecol Oncol. 2012:127(2):367-74.

31. Kim SI, et al. Impact of underweight after treatment on prognosis of advanced-stage ovarian cancer. J Immunol Res. 2014;2014:349546.

32. Vizzielli $\mathrm{G}$, et al. Does the diagnosis center influence the prognosis of ovarian cancer patients submitted to neoadjuvant chemotherapy? Anticancer Res. 2015;35(5):3027-32.

33. van Meurs HS, et al. Which patients benefit most from primary surgery or neoadjuvant chemotherapy in stage IIIC or IV ovarian cancer? An 
exploratory analysis of the European Organisation for Research and Treatment of Cancer 55971 randomised trial. Eur J Cancer. 2013;49(15): 3191-201.

34. Cornelis $\mathrm{S}$, et al. Role of neoadjuvant chemotherapy in the management of stage IIIC-IV ovarian cancer: survey results from the members of the European Society of Gynecological Oncology. Int J Gynecol Cancer. 2012; 22(3):407-16.

35. Vergote I, et al. Neoadjuvant chemotherapy is the better treatment option in some patients with stage IIIc to IV ovarian cancer. J Clin Oncol. 2011; 29(31):4076-8

36. Vergote I, et al. Neoadjuvant chemotherapy or primary surgery in stage IIIC or IV ovarian cancer. N Engl J Med. 2010;363(10):943-53.

37. Birkett R, Parry-Smith W, Todd R. Raised Ca125 and ascites: Is this ovarian cancer till proven otherwise? J Obstet Gynaecol. 2016;36(4):498-9.

38. Zivadinovic $R$, et al. Ascites in ovarian carcinoma - reliability and limitations of cytological analysis. West Indian Med J. 2015;64(3):236-40.

39. Ferriss $\mathrm{JS}$, et al. Ascites predicts treatment benefit of bevacizumab in frontline therapy of advanced epithelial ovarian, fallopian tube and peritoneal cancers: an NRG oncology/GOG study. Gynecol Oncol. 2015;139(1):17-22.

40. Cohen M, Petignat P. The bright side of ascites in ovarian cancer. Cell Cycle. 2014;13(15):2319.

41. Smolle E, Taucher V, Haybaeck J. Malignant ascites in ovarian cancer and the role of targeted therapeutics. Anticancer Res. 2014;34(4):1553-61.

42. Feldheiser A, et al. Impact of ascites on the perioperative course of patients with advanced ovarian cancer undergoing extensive cytoreduction: results of a study on 119 patients. Int J Gynecol Cancer. 2014;24(3):478-87.

43. Kehoe $\mathrm{S}$, et al. Primary chemotherapy versus primary surgery for newly diagnosed advanced ovarian cancer (CHORUS): an open-label, randomised, controlled, non-inferiority trial. Lancet. 2015;386(9990):249-57.

44. Fagotti A, et al. Phase III randomised clinical trial comparing primary surgery versus neoadjuvant chemotherapy in advanced epithelial ovarian cancer with high tumour load (SCORPION trial): Final analysis of peri-operative outcome. Eur J Cancer. 2016:59:22-33.

45. Onda T, et al. Comparison of treatment invasiveness between upfront debulking surgery versus interval debulking surgery following neoadjuvant chemotherapy for stage III/IV ovarian, tubal, and peritoneal cancers in a phase III randomised trial: Japan clinical oncology group study JCOG0602. Eur J Cancer. 2016;64:22-31.

46. Kang S. Neoadjuvant chemotherapy for ovarian cancer: do we have enough evidence? Lancet. 2015;386(9990):223-4.

\section{Submit your next manuscript to BioMed Central and we will help you at every step:}

- We accept pre-submission inquiries

- Our selector tool helps you to find the most relevant journal

- We provide round the clock customer support

- Convenient online submission

- Thorough peer review

- Inclusion in PubMed and all major indexing services

- Maximum visibility for your research

Submit your manuscript at www.biomedcentral.com/submit

) Biomed Central 